\title{
THE RADII OF STARLIKENESS AND CONVEXITY OF CERTAIN NEVANLINNA ANALYTIC FUNCTIONS
}

\author{
MAXWELL O. READE AND PAVEL G. TODOROV
}

\begin{abstract}
We determine the radius of starlikeness and the radius of convexity of analytic functions having the forms $\int_{-1}^{1} d \mu(t) /(z-t)$ and $\int_{-1}^{1} z d \mu(t) /(1-t z)$ where $\mu(t)$ is a probability measure.
\end{abstract}

1. Introduction. Let $N_{1}$ and $N_{2}$ denote the sets of all functions of the form

$$
f(z) \equiv \int_{-1}^{1} \frac{d \mu(t)}{z-t}
$$

and

$$
\phi(z) \equiv \int_{-1}^{1} \frac{z d \mu(t)}{1-t z},
$$

respectively. Here $\mu(t)$ is a probability measure. We adopt the terminology of [13] and say the functions in $N_{1}\left(N_{2}\right)$ are Nevanlinna functions of the first (second) kind defined and analytic in the $z$-plane cut along $[z \mid[-1,1]]([z \mid(-\infty,-1] \cup[1, \infty)])$. We also say that $f(z)$ and $\phi(z)$ given by (1) and (2), respectively, are associated whenever the probability measure $\mu(t)$ is the same in both formulas. Of course, $\phi(1 / z) \equiv f(z)$.

Distler [2], Dundučenko [3], Reade [6], Tchakaloff [9], [10] and Thale [11] found that the (maximal) domain of univalence for $N_{1}$ is the set $[z|| z \mid>1]$, and that for $N_{2}$ is the unit disc $\Delta \equiv[z|| z \mid<1]$. On the other hand, Todorov showed that if the measure $\mu(t)$ in (1) and (2) is strictly increasing, then each $f \in N_{1}$ is univalent for $[z|| z \mid>1] \backslash[z \mid z= \pm 1]$ and each $\phi \in N_{2}$ is univalent for $z \in(\bar{\Delta} \backslash[z \mid z= \pm 1])$ [12]-[14].

In this note, we find the radii of convexity and starlikeness of both $N_{1}$ and $N_{2}$. In addition, we find maximal domains of univalence of the set of certain normalized typically-real functions. This last is a result due to Brannan and Kirwan [1].

The results contained in this note parallel similar ones obtained by Wirths [15] for his set $T$ of all functions $g$ of the form

$$
g(z) \equiv \int_{0}^{1} \frac{z d \mu(t)}{1-t z}
$$

2. Results. Our results depend on obtaining the minimum values of certain well-known functionals. Rather than use Golusin's now-standard variational method [4], we use the following striking result due to Ruscheweyh [8].

Received by the editors April 3, 1980 and, in revised form, December 16, 1980.

1980 Mathematics Subject Classification. Primary 30C20; Secondary 30C45.

Key words and phrases. Univalence, convexity, starlikeness. 
THEOREM A. Let $g(z, t)$ be continuous on the set $\Delta x[a, b]$, and let $g$ be analytic in $z$ for each fixed $t$. Let $V$ denote the set of all functions $f$ of the form

$$
f(z) \equiv \int_{a}^{b} g(z, t) d \mu(t)
$$

where $\mu(t)$ is a probability measure, and let $V^{2}$ denote that subset of $V$ defined by those $\mu(t)$ that are step functions with at most two jumps. If $L_{1}$ and $L_{2}$ are continuous linear functionals defined on the set of all functions that are analytic in $\Delta$ (with the topology of uniform convergence on compact subsets of $\Delta$ ), and if $0 \notin L_{2}\left(V^{2}\right)$, then for each $f \in V$ there corresponds an $f_{0} \in V^{2}$ such that

$$
L_{1}(f) / L_{2}(f)=L_{1}\left(f_{0}\right) / L_{2}\left(f_{0}\right)
$$

For the special case that will lead to our first result, we take $L_{1}(f) \equiv z f^{\prime}$ and $L_{2}(f) \equiv f$, so that Theorem A yields the result

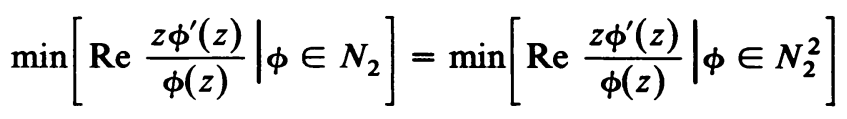

for each fixed $z, z \in \Delta$. Here $N_{2}^{2}$ is the subset of $N_{2}$ consisting of all functions $\phi$ having the form

$$
\phi(z) \equiv \frac{\gamma z}{1-t_{1} z}+\frac{(1-\gamma) z}{1-t_{2} z},
$$

where $0 \leqslant \gamma \leqslant 1,-1 \leqslant t_{1} \leqslant t_{2} \leqslant 1$. This last leads to a simple proof of the following result.

THEOREM 1. The radius of starlikeness of $N_{2}$ is

$$
r_{s} \equiv \sqrt{3(6-\sqrt{33})}=0.8753 \ldots
$$

This result is sharp. The only extremals are the functions

$$
\phi_{1}(z) \equiv \frac{(6 \pm \sqrt{6+2 \sqrt{33}}) z}{12(1+z)}+\frac{(6 \mp \sqrt{6+2 \sqrt{33}}) z}{12(1-z)}
$$

with corresponding "critical points"

$$
z=\sqrt{3(6-\sqrt{33})} e^{ \pm i \theta_{0}} \text { and } z=\sqrt{3(6-\sqrt{33})} e^{ \pm i\left(\pi-\theta_{0}\right)}
$$

respectively, where

$$
\cos \theta_{0}=\frac{1}{6} \sqrt{6(\sqrt{33}-1)}, \quad 0<\theta_{0}<\pi / 2
$$

Proof. As indicated above, we need only consider functions of the form (4) in order to solve the extremal problem (3) for fixed $z, z \in \Delta$. With that in mind, we proceed in a manner reminiscent of Kirwan [5] and Wirths [15]. A straightforward calculation using (4) gives us

$$
\frac{z \phi^{\prime}(z)}{\phi(z)}=\frac{1}{1-t_{1} z}+\frac{1}{1-t_{2} z}-\frac{1}{1-\left(\gamma t_{2}+(1-\gamma) t_{1}\right) z}
$$


If we set $z=r e^{i \theta}$ and

$$
G(s, \theta) \equiv \operatorname{Re} \frac{1}{1-s e^{i \theta}}
$$

then (9) becomes

$$
\operatorname{Re} \frac{z \phi^{\prime}(z)}{\phi(z)}=G\left(t_{1} r, \theta\right)-G\left(\left(\gamma t_{2}+(1-\gamma) t_{1}\right) r, \theta\right)+G\left(t_{2} r, \theta\right) .
$$

We now list those properties of $G(s, \theta)$ that we will need to complete our proof. Here $\theta$ is fixed, $0 \leqslant \theta<2 \pi$ and $s$ is variable, $-1 \leqslant s<1$.

(i) For $\theta=0, G(s, \theta)$ is differentiable for $-1<s<1$ and it is an increasing function there, with $G(-1,0)=1 / 2$, and $G(1,0)=+\infty$.

(ii) For $\theta=\pi, G(s, \theta)$ is differentiable for $-1<s<1$, and is a decreasing function there, with $G(-1, \pi)=+\infty$ and $G(1, \pi)=1 / 2$.

(iii) For $\theta \neq 0, \pi, G(s, \theta)$ is differentiable for $-1<s<1$ and is an increasing function for $-1 \leqslant s \leqslant s_{*}(\theta)$ for some $s_{*}(\theta)$, with $G(-1, \theta)=1 / 2$ and

$$
\max _{-1<s<1} G(s, \theta) \equiv G\left(s_{*}(\theta), \theta\right)=\frac{1+|\sin \theta|}{2|\sin \theta|} ;
$$

moreover, $G(s, \theta)$ is a decreasing function for $s_{*}(\theta)<s<1$, with $G(1, \theta)=1 / 2$. We find

$$
s_{*}(\theta)=\frac{\cos \theta}{1+|\sin \theta|}
$$

We note that (i) and (ii) can be included in (iii) if we let $s_{*}(0)=1$ with $G\left(s_{*}(0), 0\right)=+\infty$, and if we let $s_{*}(\pi)=-1$ with $G\left(s_{*}(\pi), \pi\right)=+\infty$ in (i) and (ii), respectively.

Recall that we are interested in minimizing the right-hand side of (10), where $\theta$ is fixed, $r$ is fixed, $0<r<1$, and $t_{1}, t_{2}$ and $\gamma$ are variable, with $0<\gamma<1$ and $-1 \leqslant t_{1} \leqslant \gamma t_{2}+(1-\gamma) t_{1} \leqslant t_{2} \leqslant 1$. Hence, using (i), (ii), and (iii) we can make the following assertions concerning the function

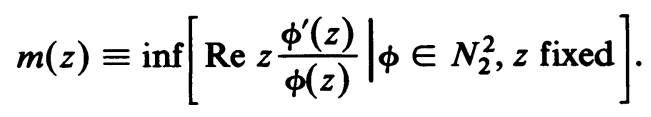

(a) If $r<s_{*}(\theta) \leqslant 1$, then $G(s, \theta)$ is increasing in the interval $-r<s<r$, so that (10) yields

$$
m(z)=\min _{-r<s<r} G(s, \theta)=G(-r, \theta)=\frac{1+r \cos \theta}{1+2 r \cos \theta+r^{2}}>0 .
$$

(b) If $-1 \leqslant s_{*}(\theta) \leqslant-r$, then $G(s, \theta)$ is decreasing in the interval $-r<s<r$, so that (10) yields

$$
m(z)=\min _{-r<s<r} G(s, \theta)=G(r, \theta)=\frac{1-r \cos \theta}{1-2 r \cos \theta+r^{2}}>0 .
$$


(c) If $-r \leqslant s_{*}(\theta)<r$, then $G(s, \theta)$ is increasing in the interval $-r<s<s_{*}(\theta)$ and decreasing in the interval $s_{*}(\theta)<s<r$, then (10) yields

$$
\begin{aligned}
m(z) & =\min _{-r<s<s_{*}(\theta)} G(s, \theta)-\max _{-r<s<r} G(s, \theta)+\min _{s_{*}(\theta)<s<r} G(s, \theta) \\
& =G(-r, \theta)-G\left(s_{*}(\theta), \theta\right)+G(r, \theta) \\
& =\frac{1+r \cos \theta}{1+2 r \cos \theta+r^{2}}-\frac{1+|\sin \theta|}{2|\sin \theta|}+\frac{1-r \cos \theta}{1-2 r \cos \theta+r^{2}},
\end{aligned}
$$

provided $\sin \theta \neq 0$.

From (a), (b), and (c) above it is evident we must use (12) to determine $r$ so that $m\left(r e^{i \theta}\right)=0$. We find

$$
r=r(\theta)=\left[\frac{(1-|\sin \theta|) \cos 2 \theta+2|\sin \theta| \sqrt{|\sin \theta|\left(\left|\sin ^{3} \theta\right|+2 \cos ^{2} \theta\right)}}{1+|\sin \theta|}\right]^{1 / 2}
$$

where $\sin 2 \theta \neq 0$. The minimum value of $r(\theta)$ in (13) is attained for

$$
|\sin \theta|=\frac{1}{6}[\sqrt{33}-3] \text {, }
$$

and this leads to the radius of starlikeness (5). (See $\$ 3$ below.)

All solutions of (14) in the interval $0<\theta<2 \pi$ are given by $\theta=\theta_{0}, \pi-\theta_{0}$, $\pi+\theta_{0}, 2 \pi-\theta_{0}$, where $\theta_{0}$ satisfies (8) and (14).

To obtain the extremal functions, we use (12); this implies that the minimum for $m(z)$ is achieved only for

$$
(1-\gamma) t_{1}+\gamma t_{2}=s_{*}\left(\theta_{0}\right) / r_{s}, \quad t_{1}=-1, t_{2}=1,
$$

and

$$
(1-\gamma) t_{1}+\gamma t_{2}=s_{*}\left(\pi-\theta_{0}\right) / r_{s}, \quad t_{1}=-1, t_{2}=1,
$$

where, by (8), (11), and (14) we have

$$
s_{*}\left(\theta_{0}\right)=\frac{1}{2} \sqrt{2(\sqrt{33}-5)}, \quad s_{*}(\pi-\theta)=-\frac{1}{2} \sqrt{2(\sqrt{33}-5)} .
$$

From (5), (15), (16), and (17) we obtain all possible extremal functions, all of which are given by (6) with corresponding "critical points" (7). This completes our proof of Theorem 1.

To obtain the radius of convexity of $N_{2}$ we use a result on typically-real functions due to Kirwan [5].

THEOREM 2. The radius of convexity of $N_{2}$ is

$$
r_{c}=(\sqrt{2}-1)
$$

which is sharp.

Proof. If we set $z=r e^{i \theta}$ in (2), then

$$
\frac{\partial}{\partial \theta} \operatorname{Re} \phi\left(r e^{i \theta}\right)=-r \sin \theta \int_{-1}^{1} \frac{\left(1-r^{2} t^{2}\right)}{\left|1-t r e^{i \theta}\right|^{4}} d \mu
$$


holds. It follows that for each $r, 0<r<1, \operatorname{Re} \phi\left(r e^{i \theta}\right)$ is decreasing for $0<\theta<\pi$ and increasing for $\pi<\theta<2 \pi$. Hence, according to a theorem due to Robertson [7], each $\phi \in N_{2}$ maps the unit disc onto a domain that is convex in the direction of the imaginary axis. Moreover, each $\phi \in N_{2}$ is real for $-1<z<1$.

Now Kirwan has shown that such functions map the disc $[z|| z \mid<(\sqrt{2}-1)]$ onto a convex domain [5].

The function $\phi_{3}(z) \equiv z /\left(1-z^{2}\right)$ shows that our result is sharp. This completes our proof.

Theorems 1 and 2 yield analogous results for the class $N_{1}$.

THEOREM 3. If $f \in N_{1}$, then $f$ maps the exterior of the unit disc onto a domain whose complement is a closed region that is convex in the direction of the imaginary axis. Moreover, the sharp radii of starlikeness and convexity of $N_{1}$ are

$$
R_{s}=1 / r_{s}=\frac{1}{3} \sqrt{\sqrt{33}+6}=1.1423 \ldots \text {, }
$$

and

$$
R_{c}=1 / r_{c}=(\sqrt{2}+1)
$$

respectively. The extremal functions are the obvious modifications of the corresponding extremals for $\mathrm{N}_{2}$.

The preceding theorems enable us to sharpen slightly some known results concerning the set $T R$ of all typically-real functions $h(z)$, where

$$
h(z) \equiv \int_{-1}^{1} \frac{z d \mu(t)}{1-2 t z+z^{2}},
$$

where $\mu(t)$ is a probability measure.

If we set $w=\frac{1}{2}(z+1 / z)$ in (18), then we obtain

$$
h(z) \equiv H(w) \equiv \frac{1}{2} \int_{-1}^{1} \frac{d \mu(t)}{w-t} .
$$

Our Theorem 3 now yields the following result.

THEOREM 4. There are two disjoint maximal domains of univalence for the class $T R$, namely

$$
D_{1} \equiv\left[z|| z+\frac{1}{z}|>2,| z \mid<1\right], \quad D_{2}=\left[z|| z+\frac{1}{z}|>2,| z \mid>1\right] .
$$

Moreover, each $h$ in TR maps

$$
D_{3} \equiv\left[z|| z+\frac{1}{z}\left|>\frac{2}{3} \sqrt{6+\sqrt{33}},\right| z \mid<1\right]
$$

and

$$
D_{4} \equiv\left[z|| z+\frac{1}{z}\left|>\frac{2}{3} \sqrt{6+\sqrt{33}},\right| z \mid>1\right]
$$

onto the complement of a starlike domain, and maps

$$
D_{5} \equiv\left[z|| z+\frac{1}{z}|>2(\sqrt{2}+1),| z \mid<1\right]
$$


and

$$
D_{6} \equiv\left[z|| z+\frac{1}{z}|>2(\sqrt{2}+1),| z \mid>1\right]
$$

onto the complement of a convex domain.

These results include the results that the images $h\left(D_{1}\right)$ and $h\left(D_{2}\right)$ are convex in the direction of the imaginary axis. We note that the domain $D_{1}$ is the intersection of the discs centered at $z= \pm i$, radius $\sqrt{2}$, and $D_{2}$ is its inverse in the circle $|z|=1$. $D_{1}$ was found by Brannan and Kirwan [1].

3. Additional details. In this section we provide most of the details that lead from (12) and (13) to (14) and (5).

First, $r(\theta)=1$ holds when $\sin \theta=0$. Second, $m\left(r e^{i \theta}\right)=0$, for $r$ given by (5) and $|\sin \theta|$ given by (14). Hence, the extremum $r_{s}$ we seek satisfies $0<r_{s}<1$.

For simplicity we set $R \equiv R(x) \equiv[r(\theta)]^{2}$, with $x \equiv|\sin \theta|$. From (12) and (13), it follows that $R(x)$, such that $m\left(r e^{i \theta}\right)=0$, satisfies

$$
(1+x) R^{2}+2\left(-1+x+2 x^{2}-2 x^{3}\right) R+(1-3 x)=0
$$

and the minimum of $R(x), 0<x<1$, occurs where $R^{\prime}(x)=0$. From (19) we obtain

$$
\frac{d R}{d x}=\frac{3+2\left(-1-4 x+6 x^{2}\right) R-R^{2}}{2\left[(1+x) R+\left(-1+x+2 x^{2}-2 x^{3}\right)\right]} \equiv \frac{N}{D}
$$

If we use (13), with $R \equiv r^{2}$, then the denominator $D$ in (20) satisfies the following:

$$
\begin{aligned}
D & \equiv \frac{1}{R}\left\{\frac{\left[\left(1-x-2 x^{2}-2 x^{3}\right)+2 x \sqrt{2 x-2 x^{3}+2 x^{4}}\right]^{2}+\left(-1+2 x+3 x^{2}\right)}{(1+x)}\right\} \\
& \geqslant \frac{1}{R} \frac{\left(1-x-2 x^{2}+2 x^{3}\right)^{2}+\left(-1+2 x+3 x^{2}\right)}{(1+x)} \equiv \frac{4 x^{3}\left(2-2 x^{2}+x^{3}\right)}{(1+x)}
\end{aligned}
$$

A simple calculation now shows that $\left(2-2 x^{2}+x^{3}\right)$, and hence $D$, is positive for $0<x<1$. Hence $\min [R(x) \mid 0<x<1]$ occurs where $R^{\prime}(x)=0$, that is where

$$
3+2\left(-1-4 x+6 x^{2}\right) R-R^{2}=0,
$$

where $R$ and $x$ also satisfy (19). From (19) and (21) we obtain

$$
\left(1+2 x-2 x^{2}-2 x^{3}\right) R=1
$$

which then combines with $(21)$ to yield $4 x^{3}(1-x)\left(2-3 x-3 x^{2}\right)=0$. This last equation leads to the value $x=|\sin \theta|$ in (14) as the $x$-value yielding the minimum of $R(x)$, and hence of $r(\theta)$. This completes our presentation of the "details" referred to above.

4. Conclusion. It would be of some interest to find the radius of convexity of the class $T R$. 


\section{BIBLIOGRAPHY}

1. D. A. Brannan and W. E. Kirwan, A covering theorem for typically-real functions, Glasgow Math. J. 10 (1969), 153-155.

2. R. J. Distler, The domain of univalence of certain classes of meromorphic functions, Proc. Amer. Math. Soc. 15 (1964), 923-928.

3. L. E. Dunducenko, A generalization of classes of analytic functions considered by L. Tchakaloff, Bulgarian Akad. Nauk Izv. Mat. Inst. 5 (1961), 35-41. (Russian, with Bulgarian and English summaries)

4. G. M. Golusin, A variational method in the theory of analytic functions, Amer. Math. Soc. Transl. 18 (1961), 1-14.

5. W. E. Kirwan, Extremal problems for the typically-real functions, Amer. J. Math. 88 (1966), 942-954.

6. Maxwell O. Reade, Two applications of close-to-convex functions, Michigan Math. J. 5 (1958), 91-94.

7. M. S. Robertson, Analytic functions starlike in one direction, Amer. J. Math. 58 (1936), 465-472.

8. St. Ruscheweyh, Nicht-lineare Extremalprobleme für holomorphe Stieltjesintegrale, Math. Z. 142 (1975), 19-23.

9. L. Tchakaloff, Sur une classe de fonctions analytiques univalentes, C. R. Acad. Sci. Paris 242 (1956), 437-439.

10. Sur les domaines d'univalence de certaines classes de fonctions analytiques, Bulgarian Akad. Nauk Izv. Mat. Inst. 4 (1960), No. 2, 43-55. (Bulgarian, with Russian and French summaries)

11. J. S. Thale, Univalence of contained fractions and Stieltjes transforms, Proc. Amer. Math. Soc. 7 (1956), 232-244.

12. P. G. Todorov, On the theory of univalent conformal mappings realizable by meromorphic functions with simple poles and pasitive residues, Ukrain. Mat. Z. 22(3) (1970), 416-422. (Russian)

13. On the theory of schlicht conformal mappings that are realized by certain Nevanlinna classes of analytic functions, Ukrain. Mat. Z. 23(1) (1971), 118-122. (Russian)

14. Maximal domains of univalence for certain classes of meromorphic functions, Aequationes Math. 11 (1974), 177-188. (Russian)

15. K. J. Wirths, Über totalmonotone Zahlenfolgen, Arch. Math. 26 (1975), 508-517.

Department of Mathematics, Universtry of Michigan, Ann Arbor, Michigan 48109

Department of Mathematics, Paissi Hilendarski University, 4000 Plovdiv, Bulgaria 